\title{
Shadow Prices for a Nonconvex Public Technology in the Presence of Private Constant Returns
}

by

\author{
John A. Weymark
}

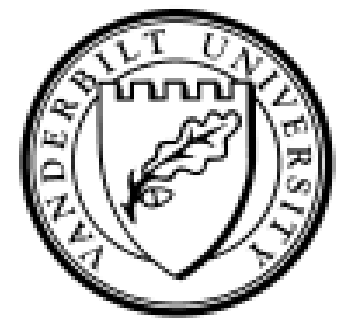

Working Paper No. 05-W01

January 2005

\section{DEPARTMENT OF ECONOMICS \\ VANDERBILT UNIVERSITY \\ NASHVILLE, TN 37235}

www.vanderbilt.edu/econ 


\title{
Shadow Prices for a Nonconvex Public Technology in the Presence of Private Constant Returns
}

\author{
by \\ John A. Weymark \\ Department of Economics, \\ Vanderbilt University, \\ VU Station B \#351819, \\ 2301 Vanderbilt Place, \\ Nashville, TN 37235-1819, USA \\ (E-mail: john •weymark@vanderbilt.edu)
}

January 2005

\begin{abstract}
Diamond and Mirrlees have shown that public sector shadow prices should be set equal to the private producer prices in some circumstances even if taxes are not optimal when the public production technology is convex and some of the private sector firms have constant-returns-to-scale technologies. In this article, it is shown that the optimal public production plan maximizes profits using the private producer prices on a subset of the public production set if this set is nonconvex. Sufficient conditions for profit maximization using these prices to identify the optimal public production plan on the whole public production set are also identified.
\end{abstract}

Journal of Economic Literature classification numbers: D61, H21.

Keywords. shadow prices, public sector pricing, Diamond and Mirrlees 


\section{Introduction}

One of the best-known results in optimal taxation theory is the production efficiency theorem of Diamond and Mirrlees (1971). This theorem shows that if commodity taxes are chosen optimally, the profits of the private sector are taxed at a $100 \%$ rate, and a mild demand regularity condition is satisfied, then the vector of optimal aggregate net outputs of the public and private production sectors is efficient. An implication of this theorem is that the appropriate shadow prices for public sector managers are the private producer prices. Hence, if the production technology of the public sector is convex, then the optimal public production maximizes profits on this production set at these prices.

However, in practice, taxes are not set optimally, which limits the applicability of the production efficiency theorem. Nevertheless, when the public technology is convex and some of the private sector firms have constant-returns-to-scale technologies, Diamond and Mirrlees (1976) have shown that public sector shadow prices should be set equal to the private producer prices in some circumstances, even if taxes are not optimal.

To state this result more precisely, it is necessary to describe the main features of the model considered by Diamond and Mirrlees. There are $n$ goods. The economy has two types of private firms: (i) profit-maximizing competitive firms with constant-returns-toscale technologies (the $C$-sector) and (ii) firms whose optimal net outputs only depend on producer prices and, possibly, on aggregate quantities. In choosing the values of its instruments, the government is assumed to choose its production plan optimally from a convex production set and it must ensure that all markets clear, but it is not constrained to set optimal taxes. Furthermore, the government is assumed to complete its transactions using the private producer prices and not to have preferences for the distribution of its revenue between taxes and receipts from its productive operations, nor for the distribution of production between sectors of the economy.

Diamond and Mirrlees' theorem, informally stated above, shows that the private producer prices are the appropriate shadow prices for the public sector if it is optimal for public production to be efficient and if the optimal net outputs of $n-1$ firms in the $C$-sector are linearly independent. Diamond and Mirrlees (1976) have also shown that in the absence of the linearly independent $C$-sector outputs, the profits of the $C$-sector firms evaluated using the public sector shadow prices are all zero, but these shadow prices need not equal the private producer prices. These two shadow pricing theorems have been shown to be special cases of more general results by Drèze and Stern (1987, Section 2.3.5), Guesnerie (1979, Section 3.2), and Guesnerie (1995, Section 4.1), to whom the reader is referred for further discussion.

One of the traditional justifications for producing goods in the public sector is the presence of significant nonconvexities in the production technologies of these goods. ${ }^{1}$ It is therefore of interest to determine if private producer prices are the correct shadow prices for the managers of the public sector in an economy in which all of Diamond and Mirrlees' assumptions (including the existence of the linearly independent $C$-sector

\footnotetext{
${ }^{1}$ See Bös (1985, Section 1) for a discussion of the various reasons for organizing production in the public sector or for publically regulating the prices at which private firms operate.
} 
outputs) are satisfied except for the convexity of the public production possibility set. In this article, I show that they are, but the scope for using these prices to decentralize the optimal public production may be limited. Specifically, the optimal public net outputs need only maximize profits using the private producer prices on a subset of the public production set. The size of this subset, which could be quite large, depends on the magnitudes of the optimal $C$-sector outputs. I also present sufficient conditions for profit maximization using these prices to identify the optimal public production plan on the whole public production set. ${ }^{2}$

In Section 2, I present Diamond and Mirrlees' shadow pricing theorems for a convex public technology. My shadow pricing theorems for a nonconvex public technology are developed in Section 3. Some concluding remarks follow in Section 4.

\section{A Convex Production Technology}

In this section, I introduce the model and present the two shadow pricing theorems for a convex public technology due to Diamond and Mirrlees (1976). In order to focus on essentials, I shall sacrifice some of the generality of their results in my presentation.

There are $n$ commodities. There are $J$ private firms, indexed by $j=1, \ldots, J$. The production possibility set of firm $j$ is $Y^{j} \subset \Re^{n}$ and its vector of net outputs is $y^{j} \in Y^{j}$. It is assumed that some private firms have constant-return-to-scale technologies. The set of such firms is $C$. Thus, $Y^{j}$ is a cone for all $j \in C$. The other private firms (if any) constitute the set $R$.

Producer prices are $p \gg 00_{n} \cdot{ }^{4}$ Each private firm is a competitive profit maximizer. At the prices $p$, the set of optimal net supplies for firm $j$ is $y^{j}(p)$. Thus, $y^{j}(\cdot)$ is the supply correspondence of the $j$ th firm. For firms in the $R$ sector, but not for firms in the $C$ sector, $y^{j}(\cdot)$ could be a function.

The government's production possibility set is $Z \subset \Re^{n}$. The vector of net outputs of the public sector is $z \in Z$. The government transacts with the private sector using the producer prices $p$. Thus, the profit (loss if negative) of the public sector from its production activities is $p z$.

It is only necessary to consider the aggregate demand behaviour of the consumer sector of the economy. Consumer prices are $q \gg 0_{n}$. The aggregate net demand vector is $x \in \Re^{n}$. This aggregate net demand vector depends on $q$ and the net (of tax) profits of the individuals in this economy. After-tax profits are functions of the producer prices $p$ and the variables $\tau$ related to profit taxation. Different interpretations of $\tau$ are possible. For example, if all firms are taxed at at common rate $\rho$, then $\tau=\rho$. Alternatively, if all profits are distributed to individuals who are then taxed on their profit income at possibly person-specific tax rates, then $\tau$ is the vector of these tax rates. It is assumed

\footnotetext{
${ }^{2}$ The techniques employed by Drèze and Stern (1987) and Guesnerie $(1979,1995)$ do not require the public production set to be convex. However, if it is nonconvex, their theorems only show that infinitesimal changes in the public production from the optimum do not increase profits evaluated using the private producer prices.

${ }^{3} \Re^{n}$ is the $n$-dimensional Euclidean space. The origin in $\Re^{n}$ is denoted by $0_{n}$.

${ }^{4}$ The following conventions are used for vector inequalities: for all $x, y \in \mathfrak{R}^{n}, x \gg y$ if $x_{i}>y_{i}$ for all $i=1, \ldots, n$ and $x>y$ if $x_{i} \geq y_{i}$ for all $i=1, \ldots, n$ and $x \neq y$.
} 
that given $q, p$, and $\tau$, the vector of aggregate net demands $x(q, p, \tau)$ is uniquely determined. Hence, $x(\cdot)$ is a function.

Market clearing requires that

$$
x(q, p, \tau)=\sum_{j=1}^{J} y^{j}+z
$$

where $y^{j} \in y^{j}(p)$ for all $j=1, \ldots, J$ and $z \in Z$. By Walras' Law, satisfaction of (1) is equivalent to requiring the government to balance its budget. Letting $T(p, \tau)$ denote the government's revenue from profit taxation, the government's budget is balanced if

$$
(q-p) x(q, p, \tau)+T(p, \tau)+p z=0 .
$$

The vector $t=q-p$ is the vector of (specific) commodity tax rates. As is standard in the optimal commodity tax literature, it is assumed that the government chooses $t$ indirectly through the choice of $p$ and $q$, rather than directly.

It is assumed that for all $z \in Z$, there is sufficient flexibility in the choice of $p$, $q$, and $\tau$ so that the market-clearing conditions (1) or, equivalently, the government budget constraint (2) is satisfied. It is not assumed that the government can necessarily choose the commodity and profit taxes optimally, although this is not ruled out either. For example, because of political constraints, some or all of the commodity taxes may be fixed during the period being considered.

From (2), it follows that for given $p$, the choice of $z$ only affects the set of $q$ and $\tau$ compatible with government budget balance through its effect on the public sector profits $p z$. Following Diamond and Mirrlees (1976), it is assumed that the $q$ and $\tau$ required for the government budget constraint to be satisfied are uniquely determined by the values of $p$ and $p z$. Hence,

$$
q=\phi(p, p z)
$$

and

$$
\tau=\psi(p, p z)
$$

for some functions $\phi$ and $\psi$.

Let $P$ denote the set of admissible producer prices. These are the producer prices that are feasible. This set may be quite restricted. For example, if the aggregate production technology in the $C$ sector is linear and $\bar{p}$ is orthogonal to every net output vector on the efficient frontier of this production set, then, up to a positive factor of proportionality, $\bar{p}$ is the only feasible producer price vector if this sector of the economy is to operate.

Substituting (3) and (4) into $x(q, p, \tau)$, we obtain a 'reduced-form' of the aggregate net demands, $\tilde{x}(p, p z)$. The market clearing condition can then be rewritten as

$$
\tilde{x}(p, p z)=\sum_{j=1}^{J} y^{j}+z
$$


where $y^{j} \in y^{j}(p)$ for all $j=1, \ldots, J$ and $z \in Z$.

Social welfare is assumed to only depend on the consumption of individuals. The social welfare function need not be individualistic in the sense of only depending on an individual's consumption indirectly through its effects on this person's utility. Taking note of (3) and (4), we can express the social welfare function in indirect form as $V(p, p z)$.

The government chooses $z \in Z, p \in P$, and $y^{j} \in y^{j}(p), j=1, \ldots, J$, to maximize $V(p, p z)$ subject to (5). It is assumed that a solution $\left(z^{*}, p^{*}, y^{1 *}, \ldots, y^{J *}\right)$ to this problem exists.

It is also assumed that any optimal public production vector $z^{*}$ is (weakly) efficient; i.e., there is no other $z \in Z$ for which $z \gg z^{*}$. It is not assumed that public production necessarily involves all commodities. As a consequence, $Z$ could have an empty interior. Efficiency implies that $z^{*}$ is on the relative frontier of $Z .^{5}$ Because $z^{*}$ is efficient, if, as Diamond and Mirrlees assume, $Z$ is convex, then $z^{*}$ can be supported by a set of shadow prices $s>0_{n}$. Hence, it is possible to decentralize production in the public sector by providing the managers of the public sector with the shadow prices $s$ and instructing them to choose $z$ to maximize profits at these prices.

Because it has not been assumed that commodity taxes are chosen optimally and that profits are taxed at a $100 \%$ rate, it is not possible to use the Diamond and Mirrlees (1971) production efficiency theorem to conclude that the public sector shadow prices should equal (up to a factor of proportionality) the optimal private sector producer prices $p^{*}$. Nevertheless, if $Z$ is convex, then at the shadow prices $s$ that support $z^{*}$, it must be the case that the value of the optimal net supplies $y^{j *}$ of any private sector firm with a constant-returns-to-scale technology must be zero. In other words, if it is feasible to transfer the proportion $\theta$ of a $C$-sector firm's production $y^{j *}$ to the public sector, then there is no change in social welfare. ${ }^{6}$

Theorem 1 (Diamond and Mirrlees (1976)). If (i) aggregate net demand depends in reduced form on producer prices and public sector profits, (ii) the public sector makes its transactions using the private sector producer prices, (iii) social welfare only depends on consumers' demands, (iv) any optimal public production is efficient, and ( $v$ ) $Z$ is convex, then for any solution $\left(z^{*}, p^{*}, y^{1 *}, \ldots, y^{J *}\right)$ to the government's optimization problem, there exists a vector of shadow prices $s>0$ such that $z^{*}$ maximizes $s z$ on $Z$ and such that

$$
s y^{j *}=0
$$

for all $j \in C$.

My statement of Theorem 1 differs from that of Diamond and Mirrlees in three respects. First, they do not require the firms in the $R$-sector to be competitive profit

\footnotetext{
${ }^{5}$ The vector $z$ is in the relative interior of $Z$ if there exists a relative neighbourhood of $z$ contained in $Z$. A relative neighbourhood of $z$ is the set formed by the intersection of a neighbourhood of $z$ in $\Re^{n}$ with the minimal hyperplane containing $Z$. If every relative neighbourhood of $z$ contains elements of both $Z$ and its complement, then $z$ is on the relative frontier of $Z$. For a more formal treatment of relative interiors and relative frontiers, see Arrow and Hahn (1971, p. 376).

${ }^{6}$ If $\theta<0$, then production is being transferred from the public sector to the $C$-sector firm.
} 
maximizers. Instead, they assume that the net supplies of these firms depend in reduced form on producer prices and public sector profits, as has been assumed for the consumer sector. Their assumption permits some $R$-sector firms to exhibit monopoly power. Second, Diamond and Mirrlees also permit social welfare to depend on the net supplies of the firms in the $R$-sector. Modifying the model in this way does not affect the conclusion that the indirect social welfare function can be written as a function of producer prices and public sector profits, which is the only feature of the social welfare function that is used in the proof of Theorem 1. All of the other results presented here remain valid with these assumptions concerning the form of the social welfare function and the behaviour of the $R$-sector firms.

Third, instead of assuming that $z^{*}$ is efficient, Diamond and Mirrlees merely assume that $z^{*}$ is on the relative frontier of $Z$. If, for example, $Z$ is bounded from below, $z^{*}$ can be on the relative frontier of $Z$ without being efficient. When this is the case, at least one of the shadow prices must be negative. The practical appeal of Diamond and Mirrlees' version of Theorem 1 depends on $z^{*}$ being efficient and, in their informal remarks, they assume that it is. ${ }^{7}$

The model employed in this shadow pricing theorem can also be extended in a number of other ways without affecting the conclusions of the theorem. For example, the model can be reformulated so as to allow for some kinds of consumption externalities, to allow for some forms of non-market clearing, and to allow for price vectors that differ between consumers (e.g., by region) or between firms (e.g., because of intermediate goods taxation). In addition, by reinterpeting the model in intertemporal terms, Theorem 1 becomes a theorem about optimal social discount rates. See Diamond and Mirrlees (1976, Section 5) for details.

While, in general, the optimal shadow prices need not equal the private sector producer prices $p^{*}$, Diamond and Mirrlees (1976, p. 45) have identified a special case in which $s$ must be proportional to $p^{*}$ and, hence, can be set equal to $p^{*}$. This special case occurs when there are $n-1 C$-sector firms whose optimal net supply vectors $y^{j *}$ are linearly independent. Diamond and Mirrlees do not state this theorem formally, nor do they provide a complete proof of this result. However, because the argument used to establish this theorem is needed in the next section to help prove Theorem 3, I include a complete proof here.

Theorem 2 (Diamond and Mirrlees (1976)). If, in addition to the assumptions of Theorem 1, there are $n-1 C$-sector firms whose optimal net supply vectors are linearly independent in the solution $\left(z^{*}, p^{*}, y^{1 *}, \ldots, y^{J *}\right)$ to the government's optimization problem, then the public sector shadow prices s are proportional to the optimal private sector producer prices $p^{*}$.

Proof. Let $C^{\prime} \subseteq C$ be a set of $n-1$ firms whose optimal net supply vectors $y^{j *}$ are linearly independent. Let $A$ be the matrix whose rows are the net supply vectors of the firms in $C^{\prime}$. By assumption, the rank of $A$ is $n-1$. By a standard theorem in linear algebra, the dimension of the kernel of the linear mapping $f$ defined by $A$ is equal to one. ${ }^{8}$ It follows from (6) that $s$ is in the kernel of $f$. Because each $C$-sector firm has a

\footnotetext{
${ }^{7}$ See, for example, Diamond and Mirrlees (1976, p. 41).

${ }^{8}$ See, for example, Zelinsky (1968, Theorem 5.3.2).
} 
constant-returns-to-scale technology, $p^{*} y^{j *}=0$ for all $j \in C^{\prime}$. Thus, $p^{*}$ is also in the kernel of $f$. Because the kernel is one-dimensional (and both $s$ and $p^{*}$ are nonzero), $s$ and $p^{*}$ must be proportional to each other.

\section{A Nonconvex Public Technology}

If $Z$ is nonconvex and the optimal public production $z^{*}$ is efficient, it may not be possible to decentralize the production of $z^{*}$ by specifying a set of public sector shadow prices and instructing the public managers to maximize profits using these prices. Nevertheless, there may exist shadow prices that serve as the correct guides for public decision-making in a neighbourhood of $z^{*}$. That is, there exists a $\delta>0$ such that $z^{*}$ maximizes shadow profits on

$$
Z_{\delta}=\left\{z \in Z \mid\left\|z-z^{*}\right\|<\delta\right\}
$$

Theorem 3 shows that if the assumptions of Theorem 2 are satisfied except for the convexity of $Z$, then the private producer prices $p^{*}$ are the correct shadow prices to use in order for $z^{*}$ to maximize shadow profits in some neighbourhood of $z^{*}$.

Theorem 3. If (i) aggregate net demand depends in reduced form on producer prices and public sector profits, (ii) the public sector makes its transactions using the private sector producer prices, (iii) social welfare only depends on consumers' demands, (iv) any optimal public production is efficient, and $(v)$ there are $n-1 C$-sector firms whose optimal net supply vectors are linearly independent in the solution $\left(z^{*}, p^{*}, y^{1 *}, \ldots\right.$, $\left.y^{J *}\right)$ to the government's optimization problem, then there exists a $\delta>0$ such that $z^{*}$ maximizes $p^{*} z$ on $Z_{\delta}$.

Proof. Let $C^{\prime}$ be a set of $n-1 C$-sector firms whose optimal net supply vectors $y^{j *}$ are linearly independent. Note that in order for these net supplies to be linearly independent, $y^{j *} \neq 0_{n}$ for all $j \in C^{\prime}$. The independence of these supply vectors also implies that there is a unique hyperplane $H$ containing all vectors of the form $z=z^{*}+\sum_{j \in C^{\prime}} \lambda_{j} y^{j *}$. (Negative $\lambda_{j}$ are permissible.) Choose $\delta>0$ so that for all $z \in H$ for which $\left\|z-z^{*}\right\|<\delta$, there exist $\lambda_{j}<1$ for all $j \in C^{\prime}$ such that $z=z^{*}+\sum_{j \in C^{\prime}} \lambda_{j} y^{j *}$.

Let $\hat{z}$ be any such vector. Because the technologies of the $C$-sector firms are cones and $\lambda_{j}<1$ for all $j \in C^{\prime}, \hat{y}^{j}=\left(1-\lambda_{j}\right) y^{j *} \in Y^{j}$. If $\hat{z} \in Z$, this transfer of production between the public and private sectors is feasible for the economy. Because profits of the $C$-sector firms are zero both before and after the transfer using the prices $p^{*}$, the revenue from profit taxation is unchanged. Aggregate net outputs are also unchanged, so the market clearing condition (5) is also satisfied. Hence, $\left(\hat{z}, p^{*}, \hat{y}^{1}, \ldots, \hat{y}^{J}\right)$ is also a solution to the government's optimization problem, where $\hat{y}^{j}=y^{j *}$ for all $j \notin C^{\prime}$. By assumption, $\hat{z}$ must be efficient. Thus, the hyperplane $H$ containing $\hat{z}$ does not intersect the relative interior of $Z$ in $Z_{\delta}$.

Because $z^{*}$ is efficient, there exists a normal $s$ to $H$ with $s>0_{n}$ for which $z^{*}$ maximizes $s^{*} z$ on $Z_{\delta}$. Because $s z=s z^{*}$ for all $z \in H$, it follows from the definition of $H$ that $s y^{j *}=0$ for all $j \in C^{\prime}$. Because the optimal net supplies of any firm in $C \backslash C^{\prime}$ 
is a linear combination of the optimal net supplies of the firms in $C^{\prime}$, it then follows that $s y^{j *}=0$ for all $j \in C$. The argument used in the proof of Theorem 2 shows that $s$ must be proportional to $p^{*}$.

The existence of the $n-1$ linearly independent optimal $C$-sector production vectors implies that it is possible to move locally in any direction from $z^{*}$ on the hyperplane $H$ defined in the proof of Theorem 3 by making a combination of feasible changes in the scale of operations of these producers and transferring the resulting production to the public sector. Whenever the resulting public production vector is feasible, it must be optimal, and so must be efficient. Thus, the nonnegative normal $p^{*}$ to $H$ serves as a vector of shadow prices for optimal public decision-making in a neighbourhood of $z^{*}$.

If there are fewer than $n-1$ linearly independent optimal $C$-sector production vectors, then there is no longer a unique hyperplane containing all vectors of the form $z=z^{*}+\sum_{j \in C^{\prime}} \lambda_{j} y^{j *}$. Any such hyperplane may contain a feasible public production arbitrarily close to $z^{*}$ that is not obtainable from a combination of feasible changes in the scale of operations of the $C$-sector producers. The argument used to establish Theorem 3 can then no longer be employed to argue that this public production vector is efficient. Hence, shadow prices defined using this hyperplane may not decentralize the optimal public production locally. For example, if there are three goods, one $C$ sector firm who produces good two using good one as an input, and the public sector produces goods two and three using good one as an input, then it is only possible to conclude that feasible changes in $z^{*}$ that are proportional to the $C$-sector firm's net supply vector are on the frontier of $Z$. In Theorem 1, convexity of $Z$ guarantees that at least one of the hyperplanes described above does not intersect the relative interior of $Z$, and so can be used to define shadow prices (which need not be proportional to $p^{*}$ ) that decentralize public production globally.

As is clear from the proof of Theorem 3, the choice of $\delta$ is bounded from above because it is only possible to scale down the production of any $C$-sector firm a finite amount before it ceases to operate. It is for this reason that it may not be possible to use the private producer prices $p^{*}$ to decentralize the optimal public production globally. Nevertheless, the neighbourhood of $z^{*}$ for which profit maximization using the prices $p^{*}$ identifies $z^{*}$ as an optimal production may be quite large. For example, suppose that there are two goods, one $C$-sector firm, and that the $C$-sector firm and the public sector firm both produce good two using good one as an input. By scaling down the production of the private firm, it is possible to transfer up to $\left|y^{1 *}\right|$ units of the input to the public firm. As it is also possible to transfer production from the public to the private sector, the reasoning used in the proof of Theorem 3 applies for any $z \in Z$ for which $0 \leq\left|z_{1}\right| \leq\left|z_{1}^{*}+y_{1}^{1 *}\right|$. The larger $\left|y_{1}^{1 *}\right|$ is, the larger the neighbourhood of $z^{*}$ for which the producer prices $p^{*}$ decentralize the optimal public production. More generally, if the size of the $C$-sector is large as measured by the distance of these firms' optimal net supply vectors from the origin, then the neighbourhood $Z_{\delta}$ on which $z^{*}$ necessarily maximizes profits at the prices $p^{*}$ is also large. This limited form of decentralization may be perfectly adequate in practice.

If the nonconvex regions of $Z$ are located close to $z^{*}$, it may well be the case that $z^{*}$ not only maximizes $p^{*} z$ on $Z_{\delta}$, it may also maximize $p^{*} z$ on all of $Z$. For example, consider replacing the public sector technology by its convex hull $\operatorname{ch}(Z)$. Suppose 
that any optimal public production in this new economy must also be efficient. If the solution to the government's optimization problem is unchanged when the public technology is enlarged in this way, it follows from Theorem 2 that $z^{*}$ maximizes $p^{*} z$ on $\operatorname{ch}(Z)$ and, hence, on $Z$, not just on $Z_{\delta}$. Thus, if the assumptions of Theorem 3 hold and the solution to the government's optimization problem is invariant to the convexification of the public sector technology, then the optimal public production $z^{*}$ can be decentralized globally using the prices $p^{*}$.

Theorem 4. If (i) aggregate net demand depends in reduced form on producer prices and public sector profits, (ii) the public sector makes its transactions using the private sector producer prices, (iii) social welfare only depends on consumers' demands, (iv) any optimal public production is efficient, $(v)\left(z^{*}, p^{*}, y^{1 *}, \ldots, y^{J *}\right)$ is a solution to the government's optimization problem when the public sector technology is either $Z$ or ch $(Z)$, and (vi) there are $n-1 C$-sector firms whose optimal net supply vectors are linearly independent, then $z^{*}$ maximizes $p^{*} z$ on $Z$.

Replacing the public technology with its convex hull relaxes the constraints facing the government. In general, one would expect this expansion of the public sector technology set to increase the optimal value of the social welfare function. However, Theorem 5 shows that if the assumptions of Theorem 2 hold when the technology is $\operatorname{ch}(Z)$ and the public production vector $z^{\circ}$ that maximizes profits on $Z$ using the producer prices $p^{*}$ in the solution to the government's optimization problem for the convexified technology is unique, then the optimal public production $z^{*}$ in $\operatorname{ch}(Z)$ is in fact in $Z$. Thus, the assumptions of Theorem 4 are satisfied and the public sector's optimal production vector for the actual technology $Z$ can be decentralized globally using the optimal private producer prices. Note that it has not been assumed that $z^{*}$ and $z^{\circ}$ are the same, nor has it been assumed that they yield the same profits with the prices $p^{*}$. Rather, these conclusions are implications of the theorem.

Theorem 5. If (i) aggregate net demand depends in reduced form on producer prices and public sector profits, (ii) the public sector makes its transactions using the private sector producer prices, (iii) social welfare only depends on consumers' demands, (iv) any optimal public production is efficient, (v) there are $n-1 C$-sector firms whose optimal net supply vectors are linearly independent in the solution $\left(z^{*}, p^{*}, y^{1 *}, \ldots, y^{J *}\right)$ to the government's optimization problem when the public sector technology is ch $(Z)$, and (vi) $z^{\circ}$ uniquely maximizes $p^{*} z$ on $Z$, then $z^{*}=z^{\circ}$.

Proof. When the public technology is $\operatorname{ch}(Z)$, the assumptions of Theorem 2 are satisfied. Hence, $z^{*}$ maximizes $p^{*} z$ on $c h(Z)$. Because $z^{*}$ is in $\operatorname{ch}(Z)$, there exists a positive integer $K$ such that for all $k=1, \ldots, K$, there exist vectors $z^{k} \in Z$ and scalars $\mu_{k}>0$ with $\sum_{k=1}^{K} \mu_{k}=1$ such that $z^{*}=\sum_{k=1}^{K} \mu_{k} z^{k}$. Because $z^{*}$ maximizes $p^{*} z$ on $\operatorname{ch}(Z)$, it then follows that $p^{*} z^{k}=p^{*} z^{*}$ for all $k=1, \ldots, K$. Thus, each of the $z^{k}$ maximizes $p^{*} z$ on $Z$. By assumption, $z^{\circ}$ uniquely maximizes $p^{*} z$ on $Z$. Hence, $K=1$ and $z^{*}=z^{1}=z^{\circ}$.

The assumption in Theorem 5 that $z^{\circ}$ uniquely maximizes $p^{*} z$ on $Z$ is essential. If, for example, $z^{*} \in \operatorname{ch}(Z) \backslash Z$ and $z^{*}$ is a strict convex combination of $z^{1}$ and $z^{2}$, both of which maximize $p^{*} z$ on $Z$, then it is possible that social welfare is lower with either 
$z^{1}$ and $z^{2}$ than with $z^{*}$ because neither $z^{1}$ nor $z^{2}$ is part of a feasible allocation with the prices $p^{*}$. In such a situation, convexifying the technology $Z$ increases social welfare, and the assumptions of Theorem 4 do not apply.

\section{Concluding Remarks}

Diamond and Mirrlees (1976, p. 45) note that a limitation of their shadow pricing theorems is that they assume that optimal public production is efficient, rather than deducing efficiency from more fundamental properties of the model. To illustrate the limitations of this assumption, they cite examples from Diamond and Mirrlees (1971) in which aggregate production efficiency is not optimal. However, even if the sum of the private and public net outputs is not on the frontier of the aggregate production possibilities set, it does not follow that public production must be inefficient. In fact, Weymark (1981) has shown that with relatively weak assumptions about the production technologies, any net output vector in the aggregate production possibilities set can be obtained as the sum of net outputs that are efficient for each firm. ${ }^{9}$ Hence, it is not restrictive to assume that public production is efficient provided that it is possible to obtain any net output vector that is efficient for the private sector in the aggregate using the available policy instruments. This will be the case if all private firms are competitive profit maximizers with convex technologies and any nonnegative producer price vector is feasible.

The theorems presented in this article for a nonconvex public technology $Z$ show that if there is enough independence in the optimal supplies of the $C$-sector firms, then the optimal public production $z^{*}$ maximizes profits using the private producer prices over some region of $Z$ containing $z^{*}$. This region may be quite large. If the solution to the government's optimization problem is invariant to the replacement of $Z$ by its convex hull, then $z^{*}$ maximizes profits at these prices on all of $Z$. Whether convexifying the public technology changes the optimal public production depends on where on the frontier of $Z$ that $z^{*}$ is located. This dependence is reminiscent of the finding by Moore et al. (1972) that simply changing the resource endowment can affect whether Pareto optimal allocations in a first-best economy with a nonconvex aggregate production technology can be decentralized as competitive equilibria. As is the case here, the circumstances that they have identified in which it is possible to decentralize Pareto optima depends on properties of a convexified version of the economy. It would be of interest to see if the techniques that Moore, Whinston, and $\mathrm{Wu}$ introduced to analyze their problem can be adapted to provide further insight into the situations in which private producer prices can be used to globally decentralize optimal public production in the kind of second-best environments considered in this article.

\footnotetext{
${ }^{9}$ His formal theorem assumes that the technologies are convex, but, as he notes, the convexity assumption is not needed for his result if the aggregate private production set and the public production set are bounded above by hyperplanes that are not parallel to each other.
} 


\section{References}

Arrow, K. J. and F. Hahn: 1971, General Competitive Analysis. San Francisco: HoldenDay.

Bös, D.: 1985, 'Public Sector Pricing'. In: A. J. Auerbach and M. Feldstein (eds.): Handbook of Public Economics, Vol. 1. Amsterdam: North-Holland, pp. 129-211.

Diamond, P. A. and J. A. Mirrlees: 1971, 'Optimal Taxation and Public Production I: Production Efficiency'. American Economic Review 61, 8-27.

Diamond, P. A. and J. A. Mirrlees: 1976, 'Private Constant Returns and Public Shadow Prices'. Review of Economic Studies 43, 41-47.

Drèze, J. and N. Stern: 1987, 'The Theory of Cost-Benefit Analysis'. In: A. J. Auerbach and M. Feldstein (eds.): Handbook of Public Economics, Vol. 2. Amsterdam: North-Holland, pp. 909-989.

Guesnerie, R.: 1979, 'General Statements on Second Best Pareto Optimality'. Journal of Mathematical Economics 6, 169-194.

Guesnerie, R.: 1995, A Contribution to the Pure Theory of Taxation. Cambridge University Press: Cambridge.

Moore, J. C., A. B. Whinston, and J. S. Wu: 1972, 'Resource Allocation in a Nonconvex Economy'. Review of Economic Studies 39, 303-323.

Weymark, J. A.: 1981, 'On Sums of Production Set Frontiers'. Review of Economic Studies 48, 179-183.

Zelinsky, D.: 1968, A First Course in Linear Algebra. New York: Academic Press. 